\title{
Upper Limb Non-Traumatic Affections in a Rural Tertiary Health Care Center
}

\author{
NS Dhaniwala* \\ Professor of Orthopedics, Datta Meghe Institute of Medical Sciences, India \\ *Corresponding author: Nareshkumar S Dhaniwala, M.S. (Ortho), Professor of \\ Orthopedics, JNMC \& AVBRH, Datta Meghe Institute of Medical Sciences, Sawangi (M), \\ Wardha 442001, M.S, India, Email: nsdhaniwala@gmail.com
}

\section{Research Article \\ Volume 3 Issue 2}

Received Date: February 25, 2019

Published Date: March 29, 2019

DOI: $10.23880 /$ jobd-16000175

\section{Abstract}

Introduction: Non-traumatic upper limb disorders are not uncommon. These may range from non-specific myalgia \& fibro-fasciitis to specific disorders of joints and bones of the upper limb bones. Frequent key board use is also becoming a new etiologic factor.

Material \& Methods: The present prospective study was done in rural tertiary health care facility on admitted cases having upper limb non-traumatic disorders. Their pre -treatment functional status was assessed using 20 items Upper Extremity Function Index (UEFI) developed by Stanford PW et al. The data was analyzed to find the frequency of various disorders.

Observations \& Results: Out of total 41 cases, male female ratio was 2.7:1. The maximum patients 19 (46.34\%) were in the age group 02 to 20 years and the least $02(4.87 \%)>$ than 60 years. The maximum cases, $20(48.78 \%)$ were of bone tumors, benign in 16 cases and malignant in 04 cases. Other pathologies noted were, rotator cuff injury, tuberculosis, peri-arthritis shoulder, tennis elbow, nerve involvement, avascular necrosis of Lunate, inflammatory arthritis and De Quervain's disease. As per the Upper Extremity Functional Index, the scores ranged from 0 to 80 . The minimum scores were seen in patients having gross neurological deficit in upper limb.

Keywords: Non-Traumatic; Upper Limb Disorders; Giant Cell Tumor; Osteochondroma

\section{Introduction}

Upper limb disorders (ULDs) are common and so are the difficulties in specific diagnosis of these disorders. These disorders may be related to muscles, tendons, joints, bony pathology, and neuropathic disorders. Complaints of arm, neck and shoulder are a major health problem in Western societies [1,2] and a huge economic burden.
In upper limb disorders, there appears an association between repetitive use of a particular muscle group or joint in the workplace and the risk of a regional pain syndrome at the site. In rural set up, the type of ULDs may vary from those in the urban areas, where use of electronic gadgets like computer, laptop and mobile phones is much more common and has become a part of day today life. Besides, computer-based work in most of the offices including banks, schools has created new 


\section{Journal of Orthopedics \& Bone Disorders}

health problems. The article is an attempt to analyze upper limb disorders seen in rural tertiary health care center situated in the central India.

\section{Material and Methods}

This prospective analytical study was carried out from July 2016 to June 2018 in department of Orthopedics of a tertiary care health facility. Approval from the Institutional Ethics Committee was obtained prior to the study. Non- traumatic cases of upper limb disorders presenting on a specific day Out Patient Department (OPD) in the week and willing to get admitted were enrolled in the study after informed consent. Detailed history was taken and physical examination of the patient was done. After clinical diagnosis, relevant X-rays were done and the patient was submitted to standard management depending on the specific diagnosis. The patients' upper limb function was noted using 'The Upper Extremity Functional Index '(UEFI). This index system utilizes 20 upper limb activities, with the normal maximum score of 80 and the minimum possible score of 0 . Each activity in the index is scored from 0 to 4 depending on the decrease in difficulty of carrying out the function, 0 denoting extreme difficulty or inability to perform activity, to the highest score 4, performing the activity without any difficulty. Total of the score gives overall functional status of the upper extremity.

\section{Observations and Results}

A total of 41 cases of upper limb non-traumatic disorders were enrolled in the study. The age of the patients ranged from 02 years to 62 years. There were 30 males and 11 females, the male: female ratio was 2.7: 1 . Nineteen, 19 (46.34\%) patients were between 02-20 years, $14(34.14 \%)$ patients between 21-40 years, 06 $(14.63 \%)$ patients between $41-60$ years and $02(4.87 \%)$ patients were >than 60 years in age. Thus, nearly half of the cases were less than 20 years of age.

Bone tumors were seen in $20(48.78 \%)$ cases, benign in $16(80 \%)$ cases and malignant in $04(20 \%)$ cases. The commonest tumors seen were Giant cell tumor and Osteochondroma seen in 03 cases each, followed by Aneurysmal bone cyst, Enchondroma \& Ossifying fibroma seen in 02 cases each. Unicameral bone cyst, Osteoid osteoma, Benign Fibrous Histioma and intra-osseous lipoma were seen in 01 case each. Malignant tumor seen were Ewing's sarcoma involving humerus in 02 cases, melanocarcinoma in 01 case and peripheral nerve sheath tumor in 01 case. Humerus was the most commonly involved bone in 08 cases, followed by radius and scapula each in 03 cases. Radius \& ulna together were involved in 02 cases.

Congenital contractures in finger \& axilla were seen in 01 case each, while 01 child had bilateral hemimelia, making congenital problems total in 03 cases (7.31\%). Rotator cuff injuries and Infective pathology were also seen in 03 cases each. Infection included 02 cases of tuberculous arthritis and 01 case of post pyogenic arthritis. Non- specific inflammatory arthritis was seen in 02 cases, involving both wrist in one case and elbow in another case. Avascular necrosis of lunate bone \& post traumatic ossification of elbow was seen in 01 case each. Peri arthritis shoulder was seen in 02 cases, while neuropathic joint, nerve thickening due to Henson's disease, clawing of fingers and brachial plexus involvement were noted in a total of 06 cases. Shoulder joint was the most commonly involved joint in 06 cases, followed by elbow joint involved in 03 cases.

As per The Upper Extremity Functional Index, the scores ranged from 0 to 80 . The minimum scores were seen in patients having gross neurological deficit in upper limb. Benign tumor presence in humerus, scapula, forearm or small bones of hand did not affect much of functions and the scores ranged from 60 to 80. Malignant tumor presence affected the function more, the pretreatment scores being in the range of 30 to 40 . Congenital contractures and infective pathologies affected the functions in a variable manner, depending on the site and severity of involvement. Congenital radial hemimelia \& contracture axilla cases' score were only 13 \& 19 respectively, while another case with finger contracture scored 65.

\section{Discussion}

Non-traumatic upper limb disorders are quite common, though most of the studies are about analysis of upper limb neurological involvement [3] and disorders related with repeated vibration to fingers as a result of frequent use of laptop and computers [4]. Occupational association of upper limb disorders has been noted in many studies and change in occupation style after a period of counselling has been found to be effective [5]. Use of keyboards for more than 4 hours continuously has been found to be associated with pain in neck, arm and tingling in upper limbs. Pain in the neck or upper limbs and sensory symptoms have been found in the nonmanual workers and associated with older age, smoking, headache, tiredness or stress. Regular keyboard use was found significantly associated with pain in the shoulders and the wrists or hands [6]. 


\section{Journal of Orthopedics \& Bone Disorders}

The upper Extremity Functional Index (UEFI) is a 20item, region specific, patient -reported outcome measure developed in 2001 [1]. It is used to measure upper extremity function in individuals with hand and upper extremity disorders. Patients rate their function on a 0 to 4 scale, where 0 indicates extreme difficulty and 4 indicates no difficulty in performing the task. The UFEI takes about 5 minutes to complete. The total score is computed by adding up individual item scores. Its validity and reliability have been tested by many authors [7].

Karen WB, et al. [8] carried out a study involving screening and physical examination in general working adult population to find out upper limb disorders. Among 6038 responders, 3152 reported upper limb symptoms and 1960 were examined. It was found that of subjects with pain, $44.8 \%$ had one or more specific soft tissue disorders. Shoulder tendinitis was noted $4.5 \%$ among men, and $6.1 \%$ among women; adhesive capsulitis $8.2 \%$ among men and $10.1 \%$ among women; lateral epicondylitis $1.3 \%$ among men and $1.1 \%$ among women; De Quervain's disease $0.5 \%$ among men and $1.3 \%$ among women; other tenosynovitis of the hand and wrist, $1.1 \%$ among men and $2.2 \%$ among women. Specific disorders tended to cluster and caused more physical signs than non -specific disorders. The present study is hospital based on admitted cases in rural set up. The sample size is small, yet gives an idea about type of pathology affecting upper limb. Tumors formed the bulk of problem, followed by neurological involvement of the limb, shoulder pathology, congenital pathology and infection. Humerus was noted to be the most commonly involved bone. Nonspecific inflammation was not seen frequently. The difference in the type of diseases could be because most of the nonspecific inflammatory conditions are treated on out- patient department basis. Multiple age group and occupation specific studies are likely to bring out true prevalence of various disorders affecting the upper limb. Besides, the present study included rural population which may have a different spectrum of problems.

\section{Conclusion}

Benign bony tumor involving humerus, scapula or radius, shoulder pathology such as adhesive capsulitis of shoulder, rotator cuff injuries, neurological problems like peripheral neuropathy, brachial plexus involvement, and congenital contractures or congenital deficiency in formation are noted to be the common non-traumatic affections of upper limb. Further age-specific and occupation specific studies involving larger population will help to know true pattern of non- traumatic affections of upper limb.

\section{References}

1. Stratford PW, Jill MB, Stratford DM (2001) Development and initial validation of the upper extremity functional index. Physiother Can 53(4): 259-267.

2. Vivian EJ Bruls, Caroline HG Bastiaenen, Rob A de Bie (2013) Non-traumatic arm, neck and shoulder complaints: prevalence, course and prognosis in a Dutch university population. BMC Musculoskel Disord 14: 8.

3. Jorgen Riis Jepsen, Carl-Goran Hagert (2010) Muscle testing in the diagnosis of work-related upper limb complaints. Europ Neurol J 2(2): 1-9.

4. Lise H Laursen, Gisela Sjogaard, Carl-Goran Hagert, Jorgen riis Jepsen (2007) Diagnostic distribution of non-traumatic upper limb disorders: vibrotactile sense in the evaluation of structured examination for optimal diagnostic criteria. La Medicina del lavoro 98(2): 127-144.

5. Burton AK, Kendall NAS, Pearce BG, Birrell LN, Bainbridge LC (2008) Management of work-relevant upper limb disorders: a review. Occupational medicine 59(1): 44-52.

6. Palmer KT, Cooper C, Walker-Bone K, Syddall H, Coggon D (2001) Use of keyboards and symptoms in the neck and arm: evidence from a national survey. Occupational Medicine 51(6): 392-395.

7. Hefford C, Abbott JH, Arnold R, Baxter GD (2012) The patient-specific functional scale: validity, reliability, and responsiveness in patients with upper extremity musculoskeletal problems. J Orthop Sports Phys Ther 42(2): 56-65.

8. Karen E Walker-Bone, Keith T Palmer, Isabel Reading, David Coggon, Cyrus Cooper (2004) Prevalence and impact of musculoskeletal disorders of the upper limb in the general population. Arthritis Care \& Research 51(4): 642-6 51. 